\title{
Los Flavonoides: Apuntes Generales y su Aplicación en la Industria de Alimentos
}

\author{
Claudia Isabel Ochoa M.* \\ Alfredo Adolfo Ayala $A^{* *}$
}

\section{RESUMEN}

Los flavonoides son compuestos fenólicos con una alta capacidad antioxidante que están

* Msc., Estudiante de Doctorado en Ingeniería, Área de Énfasis: Ingeniería de Alimentos - Facultad de Ingeniería Universidad del Valle, Santiago de Cali, Colombia.

e-mail: claochoa@univalle.edu.co

Ph.D., Profesor - Departamento de Ingeniería de Alimentos - Facultad de Ingeniería - Universidad del Valle, Santiago de Cali, Colombia.

e-mail: alfayala@univalle.edu.co

Fecha de recepción: Abril 30 de 2004

Fecha de aprobación: Diciembre 17 de 2004 presentes en la mayoría de las plantas, especialmente en las frutas y las hortalizas. Su actividad antioxidante ha atraído fuertemente la atención de las industrias de procesamiento de alimentos y de las compañías de pigmentos, cosméticas y farmacéuticas. En este artículo se presenta una revisión de literatura en cuanto a la estructura química, los procesos de extracción y cuantificación y las aplicaciones de los flavonoides.

Palabras Clave: Flavonoides, frutas, hortalizas, antioxidantes 


\section{ABSTRACT}

Flavonoids are phenolic compounds that occur in most plants. They are found basically in fruits and vegetables. Flavonoids have drawn attention in relation to their antioxidative activity. Knowledge of such compounds offers a number of opportunities in cosmetic, pharmaceutical and food processing industry. Their chemical structure, the isolation and quantification processes and their role as antioxidants are reviewed in the present work.

Key Words: Flavonoids, fruits, vegetables, antioxidants.

\section{INTRODUCCIÓN}

Las frutas y las hortalizas son fuentes ricas en vitaminas, minerales, fibra y fitoquímicos (compuestos bioactivos no nutrientes) [1]. Aunque en general todos estos componentes trabajan en forma conjunta para producir algún beneficio, los fitoquímicos, tales como los flavonoides han atraído gran interés para los productores de alimentos procesados y las industrias cosméticas y farmacéuticas debido a sus características antioxidantes.

Los flavonoides se encuentran en todas las plantas, pero están localizados principalmente en las vacuolas de las células de la piel de frutas y hortalizas [2], aportando parte del sabor y del color; la mayoría son solubles en agua y no son sintetizados por el cuerpo humano ni producidos sintéticamente. Los usos de los flavonoides dependen de su forma de combinarse; cada planta contiene una única combinación de flavonoides [3], [4], [5].

El objetivo de este trabajo es recopilar información actualizada acerca de los flavonoides, su naturaleza, propiedades, formas de extracción y aplicaciones en la industria.

\section{GENERALIDADES}

Los compuestos fenólicos, una de las clases más importantes de los fitoquímicos, se agrupan de acuerdo a su estructura química en tres grupos principales: los ácidos fenólicos, los polifenoles y Ios flavonoides, siendo este último, el grupo más común con más de 4000 compuestos identificados [1], [3]. Los flavonoides se dividen en dos grandes grupos: antocianinas $y$ antoxantinas, que se agrupan a su vez en subclases (figura 1) que están relacionadas estructuralmente pero tienen funciones diferentes[1], [5], [6], [7], [8], [9].

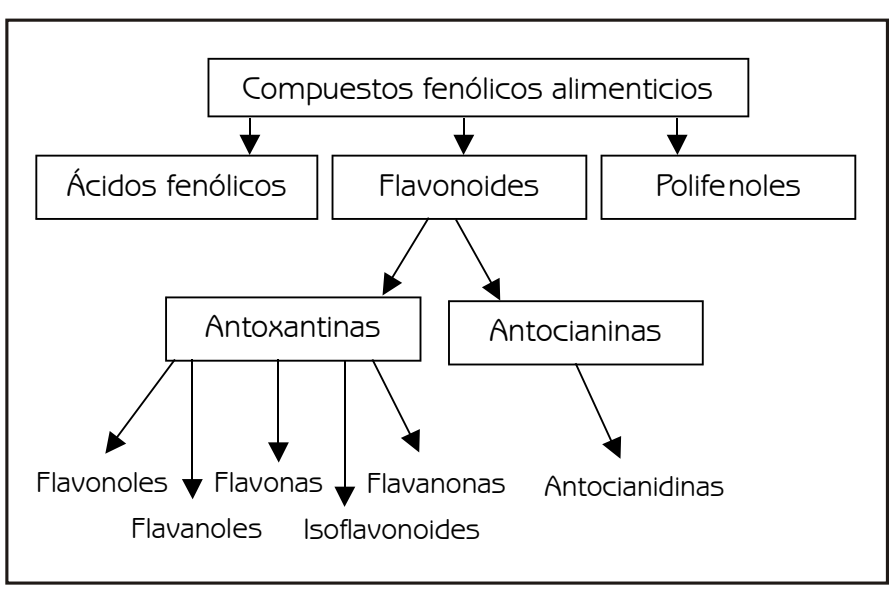

Figura 1. Árbol familiar de los compuestos fenólicos

Los flavonoides tienen una estructura de anillos formada por 15 carbonos $\left(C_{6}-C_{3}-C_{6}\right)$, que consiste en dos anillos aromáticos ( $A$ y $B$ ) que contienen generalmente grupos hidroxilos, unidos por una cadena lineal de 3 carbonos como se muestra en la figura 2. En algunos casos, el anillo heterocíclico $C$ ocurre en forma abierta [3], [10].

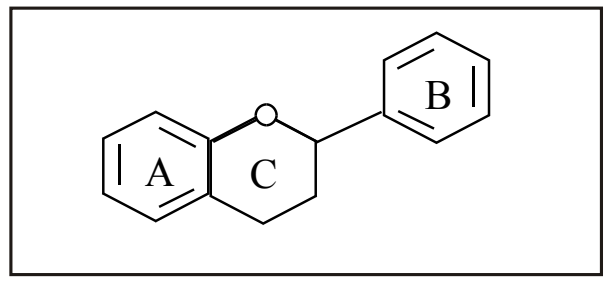

Figura 2. Estructura básica de un flavonoide 
Las subclases se categorizan generalmente por la estructura del anillo C, como se observa en la Tabla 1. La mayoría de las subclases se unen al anillo $B$ en la posición 2 del anillo heterocíclico, exceptuando a las isoflavonas, donde el anillo $B$ ocupa la posición 3. Existen muchos compuestos individuales en cada una de esas subclases que difieren en el número y la ubicación de los grupos $\mathrm{OH}$, el grado en que ellos son sustituidos y su arreglo tridimensional [3], [10].

Como se observa en la tabla 1 , los flavonoides están presentes principalmente en la cebolla, uvas, frutas cítricas, papaya, manzana, cereza, melocotón, brócoli, vino tinto, té verde $y$ productos de soya [13]. Se tienen pocos datos de otras frutas como kiwi, maracuyá, mango, piña y banano, lo cual es sorprendente debido a su significado comercial [11], aunque recientemente (1999-2000) [3] se caracterizó el kiwi y se determinó la presencia de compuestos fenólicos especialmente glucósidos y aglucones de flavonoles. También se ha comprobado que el banano es una buena fuente de antocianinas que

Tabla 1. Características de las subclases de flavonoides [11], [12]

\begin{tabular}{|c|c|c|c|}
\hline Subclase & Estructura & Compuestos & Fuente \\
\hline Flavona & & $\begin{array}{l}\text { Sinensetina, apigenina } \\
\text { Diosmina y luteolina }\end{array}$ & $\begin{array}{l}\text { Hierbas, apio, } \\
\text { pimentón, } \\
\text { uva naranja y } \\
\text { limón }\end{array}$ \\
\hline Flavonol & & $\begin{array}{l}\text { Quercetina, myricetina y } \\
\text { kaempferol }\end{array}$ & $\begin{array}{l}\text { Cebolla, vino, } \\
\text { tomate, naranja, } \\
\text { manzana, té, } \\
\text { frutas rojas }\end{array}$ \\
\hline Flavanona & & $\begin{array}{l}\text { Maringenina, hesperidina y } \\
\text { isoxanthohumol }\end{array}$ & $\begin{array}{l}\text { Miel, cítricos, } \\
\text { tomate, lúpulo y } \\
\text { cerveza }\end{array}$ \\
\hline Flavanol ó catequina & & $\begin{array}{l}\text { Catequina, epicatequina y } \\
\text { gallocatequina }\end{array}$ & $\begin{array}{l}\text { Vino, chocolate, } \\
\text { cocoa, té, uva, } \\
\text { manzana y pera }\end{array}$ \\
\hline Isoflavona & & $\begin{array}{l}\text { Genisteina, daidzeina y } \\
\text { gliciteina }\end{array}$ & $\begin{array}{l}\text { Soya y } \\
\text { leguminosas }\end{array}$ \\
\hline Antocianina & & $\begin{array}{l}\text { Cianidina, pelargonidina, } \\
\text { delfinidina y peonidina }\end{array}$ & $\begin{array}{l}\text { Frutas rojas } \\
\text { manzana, pera, } \\
\text { uva y naranja }\end{array}$ \\
\hline
\end{tabular}


contiene los seis tipos principales de éstas y además que tanto la piel como la pulpa de la guayaba son una buena fuente de antioxidantes [3].

Las tres clases de flavonoides presentes en mayor cantidad en las frutas son los flavonoles (especialmente la quecetina), las flavonas y las antocianidinas [11]. En un estudio realizado en Holanda en el año 2002 [1], el consumo total de flavonoides por persona fue de 23 mg por día, $70 \%$ del cual fue quercetina. En la Tabla 2 se presentan el contenido de quercetina en algunas frutas [1], [6].

Tabla 2. Contenido de quercetina [1]

\begin{tabular}{|c|c|}
\hline Producto & Mg /kg parte comestible \\
\hline Maranja & 41 \\
\hline Manzana & 36 \\
\hline Melocotón & 25 \\
\hline Cereza & 15 \\
\hline Uva negra & 15 \\
\hline Grosella roja & 13 \\
\hline Uva blanca & 12 \\
\hline Fresa & 9 \\
\hline Ciruela & 9 \\
\hline
\end{tabular}

\section{FUNCIÓN ANTIOXIDANTE}

La oxidación puede iniciarse por procesos físicos - químicos y continúa indefinidamente en presencia de un sustrato apropiado hasta que un mecanismo de defensa bloquee el proceso. Las sustancias objetivo son los ácidos grasos poliinsaturados, el colesterol y el DMA, pero los lípidos como constituyentes de la membrana celular son los más susceptibles debido a la velocidad de progreso de las reacciones destructivas en cadena [11].

Los radicales libres son moléculas, o porciones de ellas, con un electrón menos de lo normal. Estas moléculas son altamente reactivas y por lo tanto capturan fácilmente un átomo de otras moléculas iniciando una reacción en cadena [14]. Un radical libre se genera por la remoción del hidrógeno de un carbono adyacente a uno que porta el doble enlace en una molécula de un ácido graso insaturado (por ejemplo la bicapa lipídica de la membrana celular). Esta remoción ocurre por efecto del calor o de la luz. La molécula que contiene el radical libre reacciona fácilmente con $\mathrm{O}_{2}$ produciendo peróxido activado y éste a su vez causa el desplazamiento de un hidrógeno de otro ácido graso insaturado produciéndose hidroperóxido y un nuevo radical libre [15]. Las fuentes generadoras de radicales libres más importantes son el $\mathrm{O}_{2}$, el peróxido de hidrógeno $\mathrm{H}_{2} \mathrm{O}_{2}$ y el hidroxilo $\mathrm{OH}[14]$.

Los antioxidantes actúan como fuentes de hidrógeno y se oxidan en lugar del ácido graso protegiendo las células contra el daño de los radicales libres [14], [16]. Los antioxidantes naturales más conocidos son las vitaminas $C$ y $E$ ( $\alpha$ - tocoferol) y los carotenoides, sin embargo, se ha demostrado que los flavonoides también actúan como antioxidantes, dependiendo de su estructura molecular y de la cantidad y posición de sus grupos hidroxilos [17]. Se han encontrado flavonoides que tienen 20 veces más poder antioxidante que la vitamina $C$ y 50 veces más que la vitamina $E$ [13]. Las antocianinas tienen una estructura química única que las hace 3 ó 4 veces más potentes que la vitamina $C$ y ofrecen tanta protección como la vitamina E contra los daños oxidativos [13]. Adicionalmente, se cree que los efectos protectores de los flavonoides están asociados con la actividad antioxidante de la vitamina C y el b-caroteno [18].

Los flavonoides cítricos y las proantocianidinas extraídas de las semillas de la uva mejoran la absorción de la vitamina $C$, protegen la molécula de vitamina $C$ de la oxidación y mejoran y prolongan su funcionamiento. Por lo tanto, están involucrados en la conservación del colágeno que mantiene las células juntas formando los tejidos, previniendo el daño por radicales libres, inhibiendo la actividad enzimática y previniendo la síntesis y liberación de agentes inflamatorios que 
son funciones de la vitamina C [4], [19].

En el cuerpo humano, la contribución de los flavonoides al sistema de defensa antioxidante puede ser sustancial ya que el consumo diario de flavonoides puede estar entre 50 y 800 mg; este consumo es alto comparado con el promedio del consumo de vitamina $C$ (70 mg), vitamina $E$ (10 mg) o carotenoides (2 mg) [8], [17].

Los flavonoides con mayor poder antioxidante son: catequina, quercetina, isoxanthohumol, genisteina, naringenina [17] y el glucósido de cianidina C3G (presente especialmente en el vino tinto y las cerezas) [1]

\section{ANTECEDENTES}

Los primeros flavonoides fueron aislados e identificados en 1936 por Albert Szent-Györgyi, M.D., Ph.D., bioquímico Húngaro, quien ganó el premio Móbel por su descubrimiento de la vitamina C. Szent-Györgyi descubrió que los flavonoides mejoran la función de la vitamina $C$, aumentando su absorción y protegiéndola de la oxidación [4], [13], [19], [20]. En principio se les asignó el nombre de vitamina $P$ debido a que este grupo de nutrientes mejoran la permeabilidad de los capilares [4], [19]. A pesar de esto, los flavonoides no cumplen los requerimientos para ser considerados vitaminas ya que no son nutrientes esenciales que induzcan a síndromes de deficiencia y que se curen administrando la dosis de la sustancia en cuestión, por lo tanto el Comité de Momenclatura Bioquímica de la Sociedad Americana de Químicos Biológicos y el Instituto Americano de Mutrición recomendaron en 1950 que se eliminara el término "vitamina P" y se adoptara el término flavonoides [21].

En los últimos 15 años, ha habido una fuerte actividad investigativa dirigida hacia la identificación y extracción de flavonoides y la determinación de su actividad biológica [22]. Un ejemplo de ello son los más de 1000 estudios científicos sobre flavonoides de soya (isoflavonas) que parecen poseer propiedades que los distinguen de los otros tipos de flavonoides y otra gran cantidad de estudios acerca de los efectos de otros flavonoides [1] .

Los investigadores Howard M. Merken y Gary R. Beecher, químicos de ARS (Agricultural Research Service), están desarrollando una gran base de datos de flavonoides. Están analizando frutas y hortalizas muestreados por el Mutrient Data Laboratory, además están recopilando y evaluando datos de flavonoides publicados en la literatura científica o suministrados por la industria de alimentos. Se espera que el nuevo sistema se adopte universalmente para la estandarización en los métodos de medidas de flavonoides. El proyecto está financiado parcialmente por el instituto "Mational Heart, Lung and Blood Institute" y la fundación "Produce for Better Health Foundation" [23].

En la oficina de patentes americanas (USPTO) se encuentran registradas 195 patentes relacionadas con flavonoides entre 1976 y 2003. Es importante mencionar que solamente 20 se desarrollaron antes de 1995 y que 100 se publicaron entre los años 2000 y 2003 [24].

\section{PROCESOS DE EXTRACCIÓN}

Aunque el proceso para cada extracto es diferente, las etapas de concentración, separación y secado son comunes e independientes del compuesto de interés. Durante el proceso se utilizan temperaturas menores a $60^{\circ} \mathrm{C}$ y pH entre 2 y 5 para mantener la calidad del producto y además se evita el uso de aditivos, conservantes o inhibidores artificiales de microorganismos [15], [25].

El procedimiento de extracción tradicional consiste en homogenizar los tejidos en un medio de alcohol o alcohol acidificado para realizar la extracción, adsorber los pigmentos en una resina de intercambio iónico, separar el pigmento de la resina usando un solvente como etanol y secar si 
es necesario [16], [26], [27]. Por razones ambientales y de costos, se propone utilizar agua o un medio acuoso como medio de extracción. Este método es sencillo y práctico a nivel industrial y se obtienen flavonoides con buena solubilidad. El etanol es particularmente apropiado por ser miscible en agua en casi todas las proporciones y al no ser tóxico se acepta en un amplio rango de productos, además se evapora fácilmente reduciendo el costo de secado y puede condensarse nuevamente para reutilizarse en el proceso [25]. En la figura 3 se presenta el diagrama de bloques del proceso.

La materia prima debe macerarse o molerse antes del tratamiento en el medio de extracción y puede usarse fresca, congelada o seca. Se prefiere la muestra congelada para inducir a la formación de cristales que rompen las paredes celulares y facilitar la extracción de sustancias de la célula [25]. Una alternativa para aumentar la cantidad de producto en la extracción es la adición de enzimas, generalmente pectinolíticas, a la biomasa macerada para destruir los componentes de la pared celular y permitir la liberación de los compuestos. Aunque este método se ha aplicado en uvas y frambuesas, puede extenderse a cualquier proceso de extracción de flavonoides a partir de productos hortofrutícolas [16].

La extracción puede llevarse a cabo en cualquier forma que permita a los flavonoides migrar al medio de extracción: percolación, suspensión y/o solvatación de la materia prima en el medio de extracción. También se han sugerido extracciones altamente básicas (con hidróxido de calcio e hidróxido de sodio) y con un tratamiento posterior con ácido para precipitar los flavonoides como cristales, pero estos tienen muy baja solubilidad en agua [25]. Un método patentado recientemente en Estados Unidos utiliza agua presurizada entre 50 y 200 atm a una temperatura menor de $100^{\circ} \mathrm{C}$ [28].

También se ha propuesto realizar la extracción con $\mathrm{CO}_{2}$ supercrítico y otro solvente líquido [29]. Este proceso incluye macerar la materia prima a presiones entre 150 y 280 bar en un solvente como agua o alcohol que contenga el fluido supercrítico. La alta presión causa penetración del fluido supercrítico, se reduce su viscosidad y se incrementa la difusividad provocando su movilidad a través de las células y permitiendo extraer las sustancias a mayor velocidad y en mayor cantidad que los métodos tradicionales. Se utilizan temperaturas entre 40 y $80^{\circ} \mathrm{C}$ lo que reduce el riesgo de descomposición. Este tipo de extracción se ha utilizado en piel de uvas y de naranjas, entre otros.

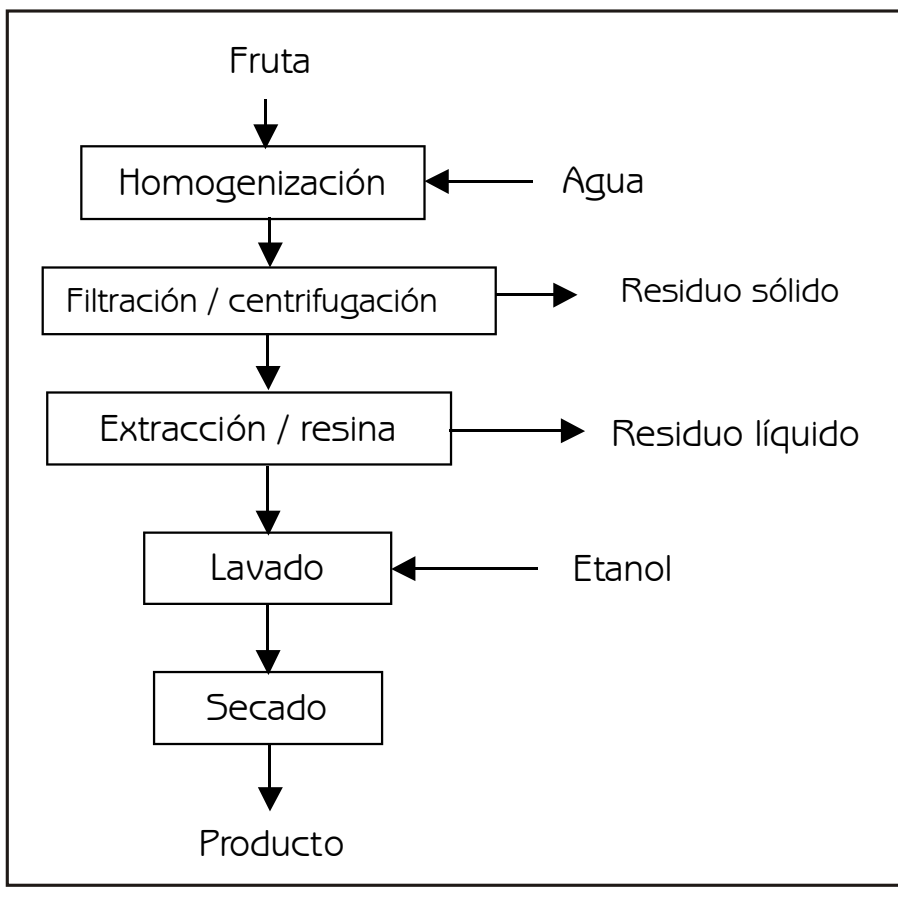

Figura 3. Diagrama de bloques de extracción de flavonoides [16], [27]

\section{CARACTERIZACIÓN}

Los métodos de caracterización e identificación de flavonoides son los mismos que se utilizan para otras sustancias naturales. El modelo más común es preparar un extracto, hacer la separación biológica y terminar con las etapas de fraccionamiento y aislamiento [11]. 
Se han desarrollado muchos métodos de cromatografía líquida para detectar y cuantificar los flavonoides en los alimentos, pero en general, sólo miden máximo tres subclases [7]. Actualmente la técnica RP-HPLC (cromatografía líquida de alto desempeño de fase inversa) es el método más popular y confiable aunque es inevitable la superposición de compuestos debido a las diferentes subclases [11]. También se han usado HPLC de fase inversa con detección electroquímica [22], resonancia magnética nuclear y cromatografía líquida con espectroscopia de masas [11]. Merken y Beecher [23], desarrollaron un sistema que encuentra y separa 18 de los flavonoides más comunes, representando las 5 subclases, que consiste en remover los azúcares mientras se separan los flavonoides del alimento contrario a lo que se hace en los métodos tradicionales.

La detección de los flavonoides se basa usualmente en absorción UV a varias longitudes de onda. La mayoría de los flavonoides muestran dos bandas principales de absorción, la primera en el rango 320-385 nm representa absorción del anillo B y la segunda en el rango $250-285 \mathrm{~nm}$ representa absorción del anillo A. El orden de elusión de los flavonoides es: glucósidos de flavanona seguidos por glucósidos de flavonol y de flavona y luego los aglicones en el mismo orden [11].

Las fases móviles que se usan generalmente son mezclas de ácido acético, agua y metanol. Aunque se han usado numerosas fases móviles con diferentes modificadores (metanol, acetonitrilo o tetrahidrofurano), ácidos (acético, fórmico o trifluoroacetico) y/o sales (fosfato de amonio) [11].

\section{ESTABILIDAD}

Tanto las antoxantinas como las antocianinas son sensibles al pH. Las antoxantinas cambian de un color amarillo en medio alcalino a blanco cremoso en medio neutro y son incoloras en medio ácido
[15], las antocianinas, a su vez, son rojas en medio ácido, moradas en medio neutro y azules en medio alcalino. Mo todos los flavonoides muestran estos cambios tan marcados en el color con el pH, esto depende de los grupos unidos a la estructura básica y a su posición [15], [16].

En cuanto al efecto de la temperatura se ha observado que las antocianinas son termolábiles (existe una relación logarítmica entre pérdida de color y temperatura) [15], [30] mientras que las antoxantinas son más estables al calor y resisten la mayoría de los tratamientos térmicos [6], [30], [31].

La alta solubilidad en agua de las antoxantinas provoca su lixiviación durante el procesamiento de frutas y hortalizas. Por otra parte, las antocianidinas son prácticamente insolubles en agua e inestables a la luz mientras que sus glucósidos (antocianinas) son más solubles y estables [16].

En general, los flavonoides reaccionan con los metales como aluminio, hierro y estaño formando complejos. Además cambian de color con iones como el sodio, el potasio, el calcio, el magnesio [15], [16]. Otro factor de inestabilidad es la presencia de enzimas que tienen carácter de $\beta$ glucosidasa ya que hidrolizan el enlace glucosídico produciendo el correspondiente aglucón que es más inestable [15].

El anhídrido sulfuroso y los sulfitos que se usan en la conservación de las frutas tienen efecto decolorante sobre los flavonoides, pues se producen formas sulfónicas que son incoloras. Esta reacción es reversible utilizando ácidos o calor [30].

Las antocianinas son inestables en presencia de oxígeno y de ácido ascórbico [16] por esto la inclusión de los flavonoides en cosméticos, productos farmacéuticos y alimentos no siempre es posible debido a la coloración oscura producida por su inestabilidad a la oxidación [32]. También 
cambian de color cuando forman complejos con otros compuestos fenólicos y en ocasiones se producen agregados poliméricos que pueden llegar a sedimentar [30].

La estabilidad de los extractos de antocianina se puede mejorar extrayendo de ellos los nutrientes tales como aminoácidos, nucleótidos y ácidos orgánicos que sirven para el crecimiento de microorganismos que pueden catalizar la oxidación. Estos materiales de bajo peso molecular se remueven por ultrafiltración o diálisis y/o resinas de intercambio iónico. Los extractos sometidos a estos procesos pueden tener mayor poder de pigmentación y ser menos higroscópicos, lo que los hace más apropiados como colorantes de productos sólidos [26]. Sin embargo, la ultrafiltración parece reducir significativamente la solubilidad de los flavonoides [25]. La estabilización de antocianinas del jugo de algunas variedades de naranja se ha logrado con adición de ácido tartárico [30]. Otro método para estabilizar los flavonoides es convertirlos en una forma estabilizada, por ejemplo un éster, esto se logra haciendo reaccionar el flavonoide con un ácido monoorgánico $\mathrm{RCOOH}$ (ácido sórbico, ascórbico, palmítico, láurico, esteárico). Este método puede usarse en forma simple, confiable y reproducible a nivel industrial [33]. También se ha propuesto hacer micro cápsulas (entre $1 \mu \mathrm{m}$ y $1 \mathrm{~mm}$ ) que están formadas especialmente de flavonoides entrecruzados [32].

\section{APLICACIONES}

\subsection{Aplicaciones en alimentos}

Los productos naturales permiten a las industrias de alimentos, de suplementos dietéticos y de aditivos de alimentos utilizar compuestos aceptados por los consumidores que actúan tan efectivamente como los productos sintéticos y ofrecen beneficios significativos a la salud [27].

\subsubsection{Antioxidantes en alimentos}

La adición de antioxidantes es uno de los métodos más populares para incrementar la vida útil de los productos alimenticios que está asociada con la peroxidación lipídica. Se ha encontrado que con antocianinas se puede lograr un porcentaje de inhibición entre 93.5 y $100 \%$, frente a los obtenidos con antioxidantes sintéticos como el BHA (87.1\%), BHT (94.3\%) y TBC (90\%) [27]. Para la producción de antioxidantes naturales se han propuesto mezclas a partir de extractos naturales de frutas que contienen antocianinas para inhibir la oxidación en varios materiales [34]. Un ejemplo de esto son los recientes estudios en estabilización de carne molida con tejidos de cereza, no sólo suprimen la peroxidación lipídica sino que inhiben la formación de aminas aromáticas heterocíclicas y la oxidación del colesterol durante el proceso de fritura [27].

\subsubsection{Pigmentos}

El uso de colorantes para lograr un color atractivo y estable de los alimentos y bebidas es indispensable, pero al igual que ocurre con los antioxidantes, la seguridad de los colorantes sintéticos está siendo cuestionada y se requiere el desarrollo de pigmentos naturales [35].

Las antocianidinas tienen gran importancia económica como pigmentos y se usan como colorantes en jugos de frutas, vino y otras bebidas para mejorar la apariencia del producto [10]. Los pigmentos de antocianinas son biodegradables y solubles en agua y no contienen toxinas ni carcinógenos y por el contrario, se sugiere que bajan el colesterol y tienen propiedades antitrombóticas y antioxidantes. Su mayor debilidad radica en la inestabilidad con los cambios de pH, calor y luz, pero su desempeño puede mejorarse realizando un proceso que se conoce como copigmentación y que consiste en adicionar algunas antoxantinas como glucósidos de flavonoides que son más estables [35].

\subsubsection{Saborizantes y edulcorantes}

Los flavonoides también se usan como sustancias saborizantes. Un ejemplo de esto es 
una mezcla de varios flavonoides de cebolla, manzana y té utilizados como sustituto de la sal en la industria alimenticia que además de ser saborizante, es antioxidante [36]. Recientemente, la naringina y la neohesperidina han adquirido mucha importancia ya que algunos de sus derivados (chalconas y dihidrochalconas) tienen un dulzor de aproximadamente 2000 veces el de la sacarosa. Se ha investigado mucho sobre estos productos por considerar que pueden ser sustitutos de los edulcorantes sintéticos. Las chalconas se obtienen por un tratamiento alcalino y cuando se reducen catalíticamente con hidrógeno, se producen las dihidrochalconas que son aún más dulces [30].

A bajas concentraciones los flavonoides, tales como los flavonoles y particularmente las catequinas se utilizan como aditivos endulzantes y/o saborizantes manteniendo por más tiempo el sabor en goma de mascar, dulces, mieles y en productos farmacéuticos como pasta dental, enjuagues bucales y drogas. Estos flavonoides mejoran el sabor y la dulzura pero no son sustitutos de endulzantes y saborizantes ya que no tienen sabor y son un poco astringentes. Parece ser que su efecto consiste en hacer más sensibles los receptores de la boca a los endulzantes lo que permite bajar los niveles de los endulzantes y saborizantes empleados [37].

\subsubsection{Alimentos funcionales}

Los alimentos y/o los ingredientes funcionales proporcionan determinados efectos fisiológicos beneficiosos no nutricionales que pueden mejorar la salud [38]. En los flavonoides se ha detectado actividad antioxidante, antiinflamatoria, anti-bacteriana y anti-cancerígena. Los productores de nutracéuticos se interesan en producir alimentos procesados estandarizados que contengan cantidades verificables de compuestos apropiados [20] con la concentración y la calidad necesarias para la prevención de algunas enfermedades [22]. Por ejemplo, el consumo regular de uvas y sus productos derivados es beneficioso para la salud, pero se pueden desarrollar productos naturales que tengan mayores concentraciones de flavonoides, utilizando los subproductos de la vinificación (cáscara y frutas) para incorporarlos posteriormente en alimentos como los cereales, los productos de panadería y confitería [38]. Otro ejemplo lo constituyen los extractos purificados de flavonoides producidos a partir de la cáscara de los cítricos que se usan como suplementos alimentarios en humanos y animales [38]. En general, los suplementos de flavonoides comerciales actualmente no contienen tan amplia cantidad de antioxidantes como los que se encuentran en una dieta natural, pero concentran los más importantes [20].

\subsection{Aplicaciones en cosméticos y farmacéuticos}

Los flavonoides son apropiados como agentes antisolares debido a que tienen una alta absorbancia UV y a que no son tóxicos. La mayor parte de la radiación UV que alcanza la tierra está entre 320 y $400 \mathrm{~nm}$ (UV-A) y aunque la radiación entre 290-320 nm (UV-B) es mucho menor, su nivel de energía es mucho mayor y causa quemaduras, daños en las células y cáncer de piel. Las flavanonas bloquean la UV-B y las flavonas son filtros particularmente efectivos para la UV-A [25], [32].

Los ésteres de flavonoides tienen aplicaciones como descongestionantes, agentes de tratamiento de piel sensible, agentes de fortalecimiento de capilares sanguíneos y agentes anti envejecimiento. Las esterasas presentes en los tejidos celulares (particularmente la epidermis), rompen los ésteres y regeneran los flavonoides iniciales, lo que permite que tengan su capacidad antioxidante inicial. Esto los hace especialmente útiles en humectantes y cosméticos [25], [33].

Por otra parte, en las pasadas dos décadas, la comunidad científica ha empezado a reconocer el valor de los productos hortofrutícolas más allá de su contribución nutriente y se han desarrollado 
anti-inflamatorios, anti-alérgicos, protectores del hígado, anti-espasmódicos, diuréticos, antibacteriales y productos para el colesterol a partir de flavonoides [1], [4], [5], [9], [17], [19], [20], [33].

Se cree que los flavonoides pueden suprimir la actividad de algunas enzimas que están involucradas en el desarrollo de enfermedades [39]. Se ha encontrado que la mayoría de los flavonoides, especialmente el quercetin son potentes y selectivos inhibidores de la enzima $\delta$ 5-lipooxigenasa que es la responsable de activar los químicos dañinos de la célula que producen cáncer [6]. El quercetin también inhibe la xantinaoxidasa (enzima que produce el ácido úrico) teniendo un significativo efecto anti-inflamatorio y la enzima aldosa reductasa que es la responsable de convertir la glucosa de la sangre en sorbitol, un compuesto fuertemente implicado en el desarrollo de la diabetes [4], [9]. Por otra parte, las flavononas producen inhibición de la fosfolipasa A2, actuando como anti-inflamatorios y las flavonas inhiben la ciclooxigenasa (COX) que está asociada con la neurodegeneración [6].

\section{CONCLUSIONES}

Debido a las propiedades antioxidantes de los flavonoides, se ha explorado su uso en aplicaciones de la industria de alimentos, cosméticos y productos farmacéuticos.

En el campo de los aditivos en alimentos hay una tendencia creciente de reemplazar los aditivos sintéticos por naturales, debido a que la población mundial está cambiando sus hábitos alimenticios prefiriendo lo natural y a las restricciones para el uso legal de antioxidantes, colorantes y productos sintéticos en alimentos por su efecto nocivo para la salud.

Existe una gran oportunidad de aprovechar los residuos generados en las industrias de procesamiento de frutas y hortalizas que contienen un alto porcentaje de flavonoides, utilizándose en la producción de colorantes, saborizantes y cosméticos.

El alto poder antioxidante de los flavonoides, se convierte en algunos casos en una debilidad debido principalmente a su inestabilidad, pero se han estudiado algunas alternativas novedosas, eficientes y a bajo costo para la solución de esta inestabilidad.

En el área de los cosméticos y farmacéuticos se ha encontrado que aunque los flavonoides no son nutrientes esenciales, actúan sobre numerosos procesos fisiológicos en el cuerpo y pueden prevenir muchas enfermedades.

\section{BIBLIOGRAFÍA}

[1]. MESSIMA, M. A Healthy Look at Sweet Cherries, Mutrition Matters, Inc. [online]. [visitado en abril, 2003 . Disponible en < http://www.nwcherries.com/metaV.html >.

[2]. AWAD, M.A., DE JAGER, A., VAM WESTIMG, L.M. "Flavonoid and chlorogenic acid levels in apple fruit: characterization of variation". Scientia Horticulturae (83), 2000, pp.249-263.

[3]. SCHIEBER, A., STIMTZIMG, F.C., CARLE, R. "Byproducts of plant food processing as a source of functional compounds-recent developments". Tends in Food Science \& Technology (12). 2001, Pp. 401-413.

[4]. Disponible en internet en <http://www. geocities.com/nutriflip/Supplements/Flavonoids. html> [visitado en abril, 2003].

[5]. Disponible en internet en <http://www. gnc.com/health_notes/Supp/Flavonoids.htm> [visitado en abril, 2003].

[6]. GRAMT, G. Excitotoxins, The Special Role of Flavonoids [online]. [visitado en abril, 2003]. Disponible en <http://www.academyofwellness. com/ excitotoxins flavonoids.htm $>$.

[7]. MERKEM H. M., BEECHER G. R. Measurement of food flavonoids: a review [online]. Tektran, United States Department of Agriculture, August, 1999 [visitado en abril, 2003]. Disponible en $<$ http://www.nal.usda.gov/ttic/tektran/data/000 010/44/0000104445.html>.

[8]. PETERSOM J., DWYER J. Flavonoids: dietary occurrence and biochemical activity [online] . 
Tektran, United States Department of Agriculture, September, 2000 [visitado en abril, 2003]. Disponible en <http://www.nal.usda. gov/ttic/tektran/data/000011/50/0000115068. $\underline{\mathrm{html}>}$.

[9]. ZAMBRAMO, L., ROJAS M y RAMÍREZ, B. Ecological significance of pigment variations in mistletoes[online]. Departamento de Biología, Universidad del Cauca, Colombia [visitado en abril, 2003 ]. Disponible en $<$ http://www.science.siu.edu/parasiticplants/Meetings/ParPapers.other.html>.

[10]. FRIEDLI, G. Friedli Enterprises [online]. [visitado en abril, 2003]. Disponible en <http://www.friedli.com/herbs/ phytochem/flavonoids.html>.

[11]. ROBARDS, K., PREMZLER, P., TUCKER, G., SWATSITAMG, P., GLOVER, W. "Phenolic compounds and their role in oxidative processes in fruits". Food Chemistry (66). 1999, pp. 401436.

[12]. Scientific Reseach [online]. [visitado en abril, 2003]. Disponible en <http://www. Chocolateinfo.com/sr/sr article 13.jsp $>$.

[13]. BERKOFF, M., Focus on Flavonoids [online]. [visitado en abril, 2003]. Disponible en

$<$ http://www. healthwell.com/hnbreakthroughs/s ep98/flavonoids. cfm?path $=$ hw $>$.

[14]. Free Radicals [online]. [visitado en abril, 2003]. Disponible en <http://www. dietaweb.it/English/radicaliE.html>.

[15]. CHARLEY, H. TECMOLOGÍA DE ALIMENTOS. PROCESOS FÍSICOS Y QUÍMICOS EM LA PREPARACIÓN DE ALIMENTOS. Ed. Limusa, México, 1999, pP. 325-328, 652-659.

[16]. GARCIA, M., QUIMTERO, R., LÓPEZ-MUMGUÍA A. BIOTECMOLOGÍA ALIMENTARIA. Ed. Limusa, México, 2000, pp. 493-494, 510.

[17].MIRAMDA, C. Antioxidant Activities of Flavonoids [online]. Department of Environmental and Molecular Toxicology Oregon State University. [visitado en abril, 2003]. Disponible en $<$ http://lpi.oregonstate.edu/fw00/flavonoid.html>.

[18]. Disponible en internet en $<$ http://www.raysahelian.com/flavonoids.html> [visitado en abril, 2003].

[19].HAAS, E. M. Excerpted from Staying Healthy with Mutrition: The Complete Guide to Diet and Mutritional Medicine [online]. [visitado en abril, 2003]. Disponible en <http://www.bpcr.com/ diet/bio-flavonoids.html>.
[20].CHALLEM, J., The Power of Flavonoids. Antioxidant Mutrients in Fruits, Vegetables, and Herbs, [online], May 1998 [visitado en abril, 2003]. Disponible en <http://www.thenutritionreporter. com/power of flavonoids.html $>$.

[21].EXQUIM, S.A. [online], [visitado en abril, 2003]. Disponible en $<$ http:/www.intselchimos. com/citrus\%20soluble\%20extract.pdf > .

[22].MUSAMI, P. Separation and Quantitation of Selected Flavonoids in Fruit Extracts by Reversed Phase -HPLC, followed by Coularray Electrochemical Detection [online], [visitado en abril, 2003]. Disponible en <http://www.rx.uga. edu/main/home/cmomany/Public/pbsseminarsp ring2002 files.htm>.

[23].MERKEN, H. M., BEECHER, G. R. Finessing the Flavonoids Agricultural Research. [online], February, 2001 [visitado en abril, 2003]. Disponible en <http://www.ars.usda.gov/ is/AR/archive/feb01/flav0201.htm>.

[24]. Oficina de Patentes Américanas [online], [visitado en abril, 2003]. Disponible en $<$ http:/www.uspto.gov>.

[25].PLASCHKE, K. Composition comprising one or more flavonoids, method of obtaining such composition and use thereof as UV-absorbing agent. United States Patent, 6,409,996, June 25, 2002.

[26]. HILTOM, B. W., LIM, R. I., TOPOR, M.G. Processed anthocyanin pigment extracts. United States Patent, 4,320,009, March 16, 1982.

[27].MURALEEDHARAM, G., MAIR, O. Method and compositions producing cherry derived products. Board of Trustees of Michigan State University. United States Patent Mo. 6,423,365, Jul. 23, 2002.

[28].WAI, C. M., LAMG, Q. Pressurized water extraction United States Patent 6,524,628, February 25, 2003.

[29].PIQUER, M. B., FERRI, B. E. M., FUEMTES, J. G., FORTEA, G. F., HUERTA, S. S. Procedure to extract natural products. United States Patent Mo.6,326504, December 4, 2001.

[30].BADUI, S. QUÍMICA DE LOS ALIMEMTOS. Pearson Education, México, 1999, pp. 388-396.

[31].EWALD, C., FJELKMER-MODIG, S., JOHAMSSOM, K., SJÖHOLM, I., ÅKESSOM, B. "Effect of processing on mayor flavonoids in processed onions, green beans, and peas". Food Chemistry (64). 1999, PP. 231-235.

[32].LEVY, M., AMDRY, M. Microcapsules with a wall of 
crosslinked plant polyphenols and compositions containing them. United States Patent, 5,780,060, July 14, 1998.

[33].PERRIER, E., MARIOTTE, A., BOUMENDJEL, A., BRESSOM-RIVAL, D. Flavonoid esters and their use notably in cosmetics. United States Patent, 6,235,294, May 22, 2001.

[34].GRAY, J. I., BOOREM, A. M., MAIR, M. G., STRASBURG, G. M., WAMG, H., GOMAA, E. A., CHAMG, Y. Method and compositions for inhibiting oxidation. United States Patent, 5,985,636, November 16, 1999.

[35].LEMOBLE, R., RICHHEIMER, S. L., BAMK, V. R., BAILEY, D. T. Pigment composition containing anthocyanins stabilized by plant extracts. United States Patent, 5,908,650, June 1, 1999.

[36].KURPPA, L. Flavoring composition, production and use thereof. SLK Foundation, Finlandia. United States Patent Mo. 6,495,185, Dec. 17, 2002.

[37].KASHKET, S. Sweetness and flavor enhanced compositions and method of preparing such compositions. United States Patent, 4,16,480, March 6, 1990.

[38].MAZZA, G. ALIMEMTOS FUMCIOMALES, ASPECTOS BIOQUIMICOS Y DE PROCESADO. Acribia S.A, España, 2000, pp. 141-231.

[39].Flavonoids in Fruits, Vegetables May Lower Lung Cancer Risk [online], Feb, 2000 [visitado en abril, 2003]. Disponible en <http://www.cancer.org/ docroot/NWS/content/NWS 1_1x Flavonoids Ma y_LowerLung_Cancer_Risk.asp>. 